\title{
Detection of Extracellular Proteases from Microorganisms on Agar Plates
}

\author{
Alane Beatriz Vermelho/ ${ }^{+}$, Maria Nazareth Leal Meirelles*, Andréa Lopes, \\ Simone Dias Gonçalves Petinate, André Adriano Chaia, \\ Marta Helena Branquinha
}

\begin{abstract}
Departamento de Microbiologia Geral, Instituto de Microbiologia, Universidade Federal do Rio de Janeiro, Cidade Universitária, Ilha do Fundão, 21944-970 Rio de Janeiro, RJ, Brasil *Laboratório de Ultraestrutura e Biologia Celular, Instituto Oswaldo Cruz, Av. Brasil 4365, 21045-900 Rio de Janeiro, RJ, Brasil
\end{abstract}

We present herein an improved assay for detecting the presence of extracellular proteases from microorganisms on agar plates. Using different substrates (gelatin, BSA, hemoglobin) incorporated into the agar and varying the culture medium composition, we were able to detect proteolytic activities from Pseudomonas aeruginosa, Micrococcus luteus and Serratia marcescens as well as the influence that these components displayed in the expression of these enzymes. For all microorganisms tested we found that in agar-BHI or yeast extract medium containing gelatin the sensitivity of proteinase detection was considerably greater than in BSA-agar or hemoglobin-agar. However, when BSA or hemoglobin were added to the culture medium, there was an increase in growth along with a marked reduction in the amount of proteinase production. In the case of $\mathrm{M}$. luteus the incorporation of glycerol in BHI or yeast extract gelatin-agar induced protease liberation. Our results indicate that the technique described here is of value for detecting extracellular proteases directly in the culture medium, by means of a qualitative assay, simple, inexpensive, straight forward method to assess the presence of the proteolytic activity of a given microorganism colony with great freedom in substrate selection.

Key words: protease - detection - microorganism

Proteases play a crucial role in numerous pathologic processes. Arthritis, tumor invasion and metastasis, infections and a number of degenerative diseases have been linked with the involvement of one or more proteolytic enzymes (Brown 1994). Microbial proteases have been proposed as virulence factors in a variety of diseases caused by microorganisms. The virulence of Pseudomonas aeruginosa is multifactorial, but it is partly determined by exoproducts such as alkaline protease and elastase, which are responsible for the damage of tissues by degrading elastin, collagen and proteoglycans. These enzymes have been also shown to degrade proteins that function in host defense in vivo (Sakata et al. 1993).

In bacteria, serine- and metalloproteases are the principal classes of proteases found in several species such as Bacillus subtilis, B. amyloliquefaciens, Pseudomonas sp., Lysobacter enzymogenes and Escherichia coli (Fujishige et al. 1992). Identification and characterization of microbial proteases are prerequisites for understanding their role in the

This work was supported by CNPq (PADCT/RHAE), CEPG/UFRJ, FINEP, FUJB and FIOCRUZ.

${ }^{+}$Corresponding author. Fax: +55-21-270.8793

Received 31 October 1995

Accepted 25 July 1996 pathogenesis of infectious diseases as well as to improve their application in biotechnology. For this aim, rapid and sensitive techniques for the detection and characterization of microbial proteases are highly desirable (Lantz \& Ciborowski 1994).

Proteases are also one of the highest value commercial enzymes. They find application in the food, pharmaceutic and detergent industries and are important tools in studying the structure of proteins and polypeptides (Bhosale et al. 1995).

The present study describes a qualitative method for detection of extracellular proteases on agar plates and the proper culture medium for their production.

\section{MATERIALS AND METHODS}

Microorganisms - The microorganisms employed in this study - P. aeruginosa ATCC 19429, Serratia marcescens isolated from clinical material at Hospital Clementino Fraga Filho, UFRJ, RJ and Micrococcus luteus isolated from skin - were maintained either in $5 \%(\mathrm{w} / \mathrm{v})$ brain heart agar (Merck) (medium A) or in $2 \%$ (w/v) sucrose (Merck), $0.5 \%$ (w/v) yeast extract (Biobrás), 2\% $(\mathrm{w} / \mathrm{v})$ peptone $(\mathrm{BBL}), 2 \%(\mathrm{w} / \mathrm{v}) \mathrm{KCl}$ (Reagen) and $1.5 \%$ (w/v) agar (Difco) (medium B).

Detection media - The detection media were the same described above, supplemented and autoclaved with $1 \%(\mathrm{w} / \mathrm{v})$ gelatin (Merck). The me- 
dium with $1 \%(\mathrm{w} / \mathrm{v})$ bovine serum albumin (BSA - Sigma) was made from a stock solution containing $1 \mathrm{~g}$ BSA made up to $5 \mathrm{ml}$ with water. This solution was sterilized using $0.22 \mu \mathrm{m}$ filter membranes and added to culture medium at $45^{\circ} \mathrm{C}$. The medium with $1 \%$ hemoglobin (BBL) was made from a stock solution containing $1 \mathrm{~g}$ hemoglobin made up to $50 \mathrm{ml}$ with water. This solution was autoclaved, cooled to approximately $50^{\circ} \mathrm{C}$ and an equal proportion to sterile medium A or B was added. Each medium was adjusted to $\mathrm{pH}$ 7.0.

Twenty $\mathrm{ml}$ of culture media A or B (supplemented with gelatin, BSA or hemoglobin) were then poured per plate and allowed to harden. A loopful of the culture growth was placed in the center of the agar plates. After inoculation, the plates were incubated at $37^{\circ} \mathrm{C}$ and observed daily for ten days. For BSA and gelatin, extracellular protease detection was done after staining with $0.1 \%$ amido black in methanol-acetic acid-water 30:10:60 (v/v/v) for $1 \mathrm{hr}$ at $28^{\circ} \mathrm{C}$. Coomassie blue $(0.25 \%$, w/v) in methanolacetic acid-water 5:1:4 (v/v/v) could also be used, although destaining may require the use of methanol and acetic acid. Regions of enzyme activity were detected as clear areas, indicating that hydrolysis of the substrates had occurred. For hemoglobin, the extracellular protease detection was directly observed in agar plates as well as with amido black and/or Coomassie blue. The proteinase activity was scored as grade - when no visible halo was present, grade + when visible proteolysis was limited to 1-2 $\mathrm{mm}$ around the colony and grade ++ when the zone of proteolysis was more than $2 \mathrm{~mm}$ from the margin of the colony.

Influence of the medium composition (carbon source) on the expression of extracellular proteases by $M$. luteus - M. luteus was inoculated in four different culture media, using $1 \%(\mathrm{w} / \mathrm{v})$ gelatin as substrate por proteolytic detection: medium A; medium B; medium C was the same as medium B with sucrose being replaced by $2 \%(\mathrm{w} / \mathrm{v})$ glycerol; medium $\mathrm{D}$ with the following composition: $2 \%(\mathrm{w} /$ v) glycerol (Reagen), $0.45 \%(\mathrm{w} / \mathrm{v})$ peptone, $0.2 \%$ $(\mathrm{w} / \mathrm{v}) \mathrm{K}_{2} \mathrm{HPO}_{4}, 0.03 \%(\mathrm{w} / \mathrm{v}) \mathrm{KCl}, 0.05 \%(\mathrm{w} / \mathrm{v})$ $\mathrm{MgSO}_{4}$ and $1.5 \%$ agar.

\section{RESULTS}

Effect of protein substrates and culture media in extracellular protease production - M. luteus, $S$. marcescens and $P$. aeruginosa were screened for extracellular proteinase production by inoculation onto BHI- or yeast extract-agar medium supplemented with gelatin, BSA or hemoglobin as substrate. Protease activity was detected $24 \mathrm{hr}$ postinoculation. Clear halos increased until the fifth day, and remained constant during ten days. All microorganisms tested hydrolysed preferentially gelatin incorporated in BHI or yeast extract. When the culture medium was supplemented with BSA or hemoglobin, the microorganisms caused proteolysis beyond the hinge of colonies or just a slight hydrolysis (Table).

$M$. luteus hydrolysed gelatin in BHI-agar, but exhibited no zones of clearing in yeast extract-agar (Fig. 1). This bacterium did not hydrolyse BSA in both culture media (Fig. 2), but utilize hemoglobin producing clear zones only around the colonies (Fig. 3).

$P$. aeruginosa showed great clear halos in gelatin-media (Fig. 1). However, when inoculated in BSA- and hemoglobin-agar in both media, a very low production of extracellular proteases was detected, although the growth of the colonies was increased (Figs 2, 3).

The microorganism $S$. marcescens also hydrolysed preferentially gelatin (Fig. 1) but did not produce detectable proteolytic enzymes in hemoglobin medium, which was the best medium for the growth of this bacterium (not shown). It also yields low proteolytic activity in BSA-containing media (Fig. 2).

Effect of the carbon source on the expression of extracellular proteases by M. luteus - Yeast extract supplemented with sucrose inhibited the protease secretion by $M$. luteus. Using the same culture medium but replacing sucrose by glycerol as carbon source a marked increase in protease production was observed. The same effect was observed when the glycerol-containing medium D was used (Fig. 3).

TABLE

Influence of proteins substrates (gelatin, hemoglobin and BSA) and the culture media (yeast extract and BHIagar) in the production of extracellular proteases (for experimental details see Materials and Methods)

\begin{tabular}{lccc}
\hline & $\begin{array}{c}\text { Micrococcus } \\
\text { luteus }\end{array}$ & $\begin{array}{c}\text { Pseudomonas } \\
\text { aeruginosa }\end{array}$ & $\begin{array}{c}\text { Serratia } \\
\text { marcencens }\end{array}$ \\
\hline Gelatin-yeast extract & - & ++ & ++ \\
Gelatin-BHI & ++ & ++ & ++ \\
Hemoblobin-yeast extract & + & ++ & - \\
Hemoglobin-BHI & + & ++ & - \\
BSA-yeast extract & - & + & + \\
BSA-BHI & - & + & + \\
\hline
\end{tabular}


A
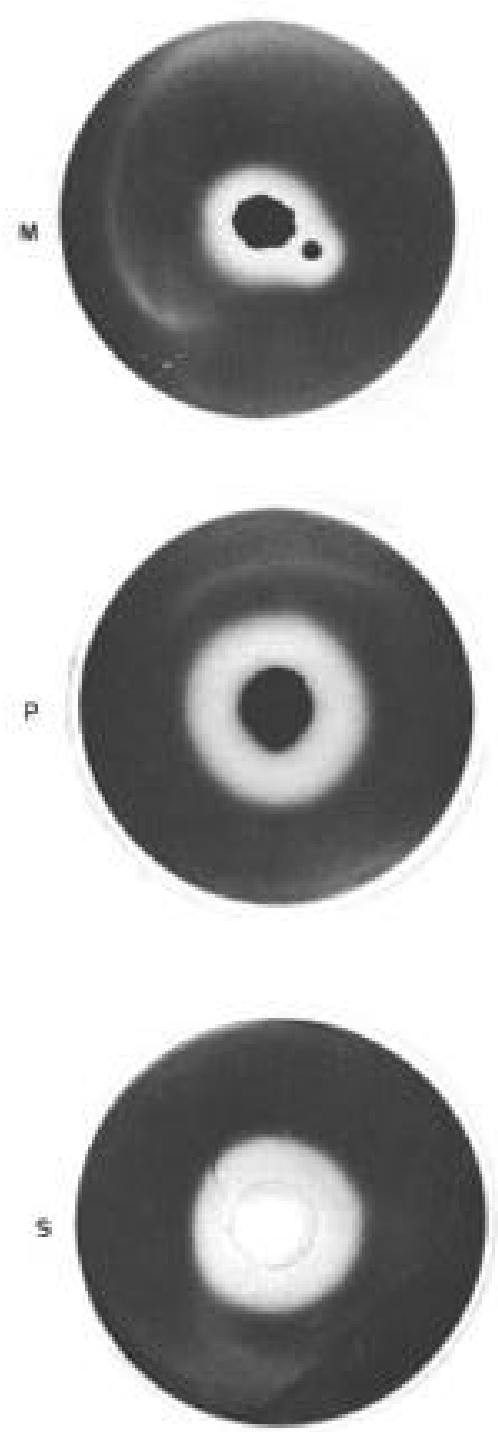

B
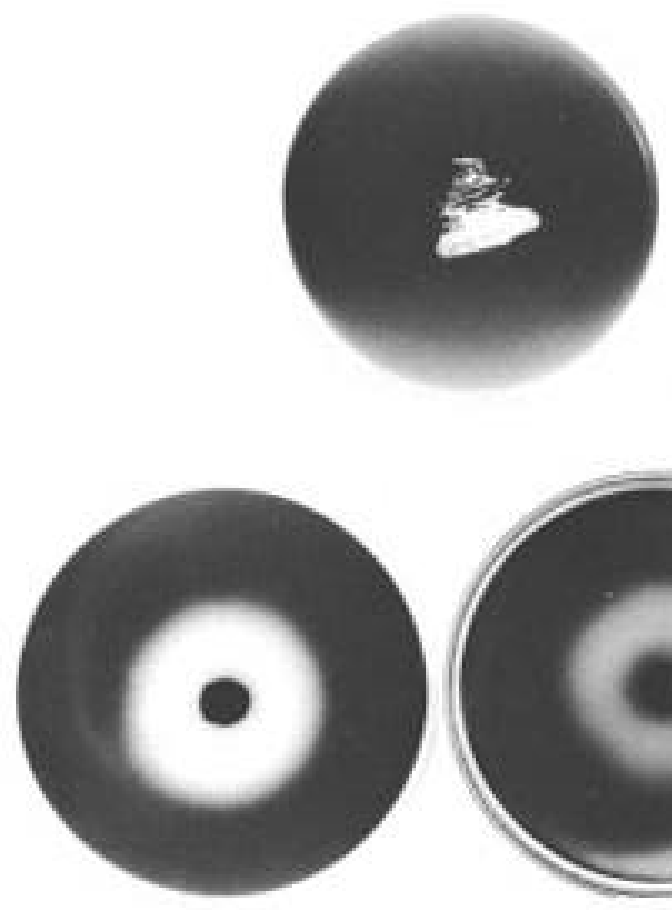

1
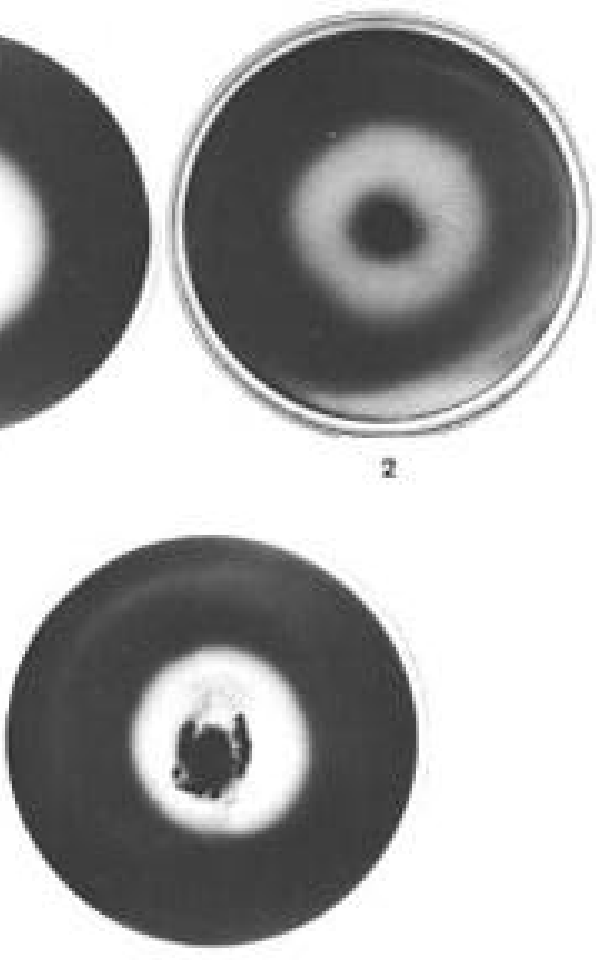

Fig. 1: proteolytic activity on agar plates containing gelatin as substrate and $\mathrm{A}, \mathrm{BHI}-\mathrm{agar}$ and $\mathrm{B}$, yeast extract-agar as nutrient media. All plates were stained with amido black, except B2 that was stained with Coomassie blue. M: Micrococcus luteus, P: Pseudomonas aeruginosa, S: Serratia marcescens.

\section{DISCUSSION}

This study has shown that protein substrates can influence markedly the production of extracellular proteases by bacteria such as $P$. aeruginosa, S. marcescens and M. luteus. There was also evidence which indicated that the composition of the medium can modify the influence of a substrate on protease production.

The most proeminent proteolytic activity was found in $P$. aeruginosa, which hydrolysed gelatin, BSA and hemoglobin. It is known that $P$. aeruginosa secrete two metalloendopeptidases: the elastase, now called pseudolysin (EC 3.4.24.26) and an alkaline protease (Morihara 1995). S. marcescens hydrolysed preferentially gelatin and did not utilize hemoglobin as protein substrate. This microorganism produces two metalloproteinases with $\mathrm{Mr}$ of $56 \mathrm{kDa}$ and $60 \mathrm{kDa}$, being the $56 \mathrm{kDa}$ the proeminent enzyme and a $73 \mathrm{kDa}$ thiol-dependent proteinase (Maeda \& Morihara 1995). The bacterium M. luteus did not use BSA as substrate on agar plates and produced a weak proteolytic activity when hemoglobin was incorporated in the nutrient agar plates. The best substrate for protease production for M. luteus was gelatin. Preliminary studies of our laboratory showed that it produces a high-molecular weight serine-protease (Petinate et al. 1995). 
A

M
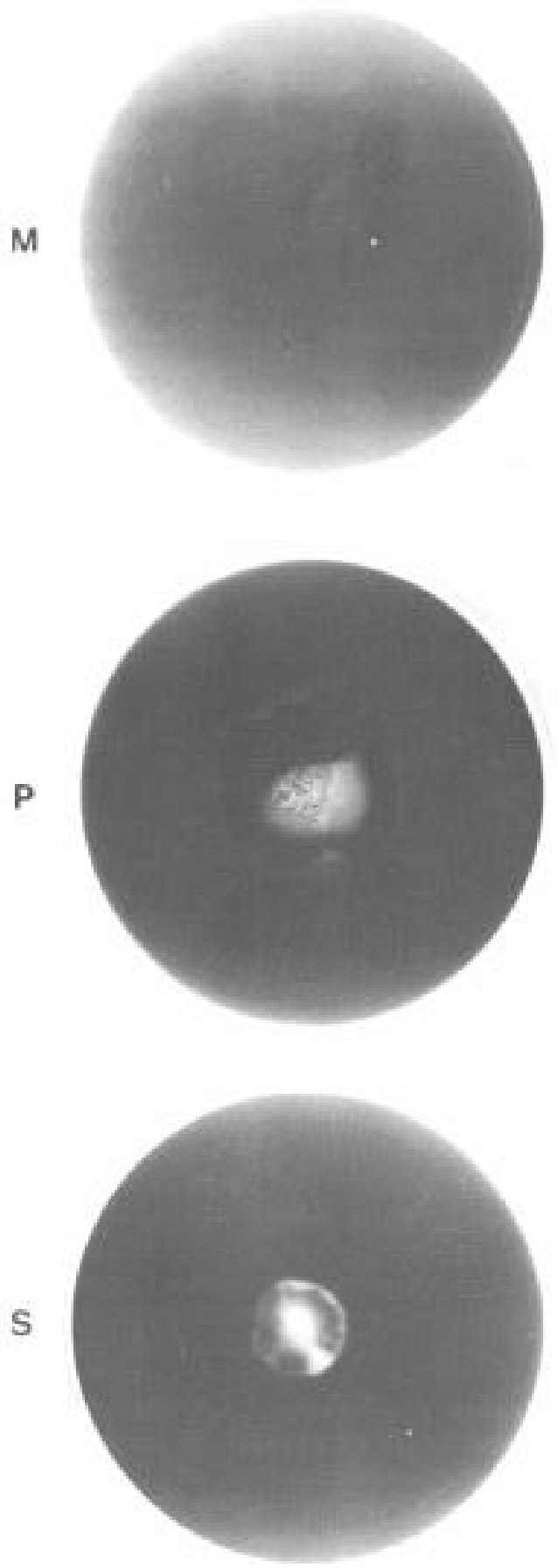

B
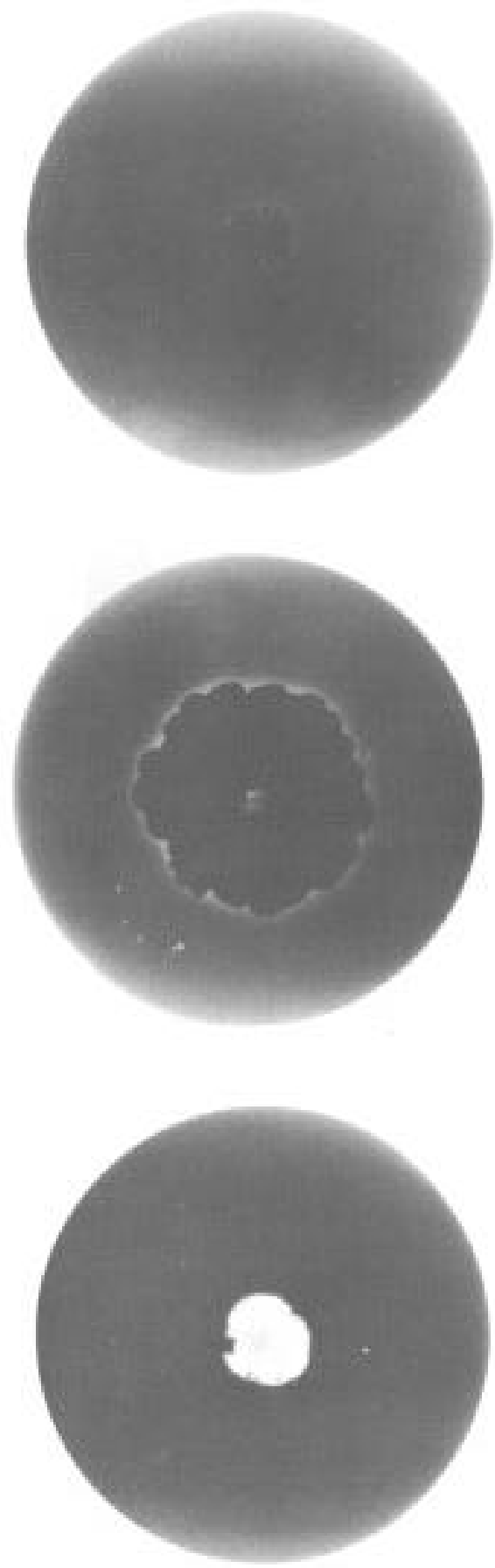

Fig. 2: production of extracellular proteases by Micrococcus luteus (M), Pseudomonas aeruginosa $(\mathrm{P})$ and Serratia marcescens (S) on agar plates containing BSA as substrate and A, BHI-agar and B, yeast extract-agar as nutrient media.

Differences in the ability to utilize gelatin, hemoglobin and BSA may be due to differences in the substrate specificities of the enzyme produced (de Giorgi et al. 1985, Ponsare et al. 1985) or may be the consequence of parameters known to affect extracellular proteinase production, such as $\mathrm{pH}$, medium composition and temperature. Gelatin, a type 1 collagen, was the most effective protease- 

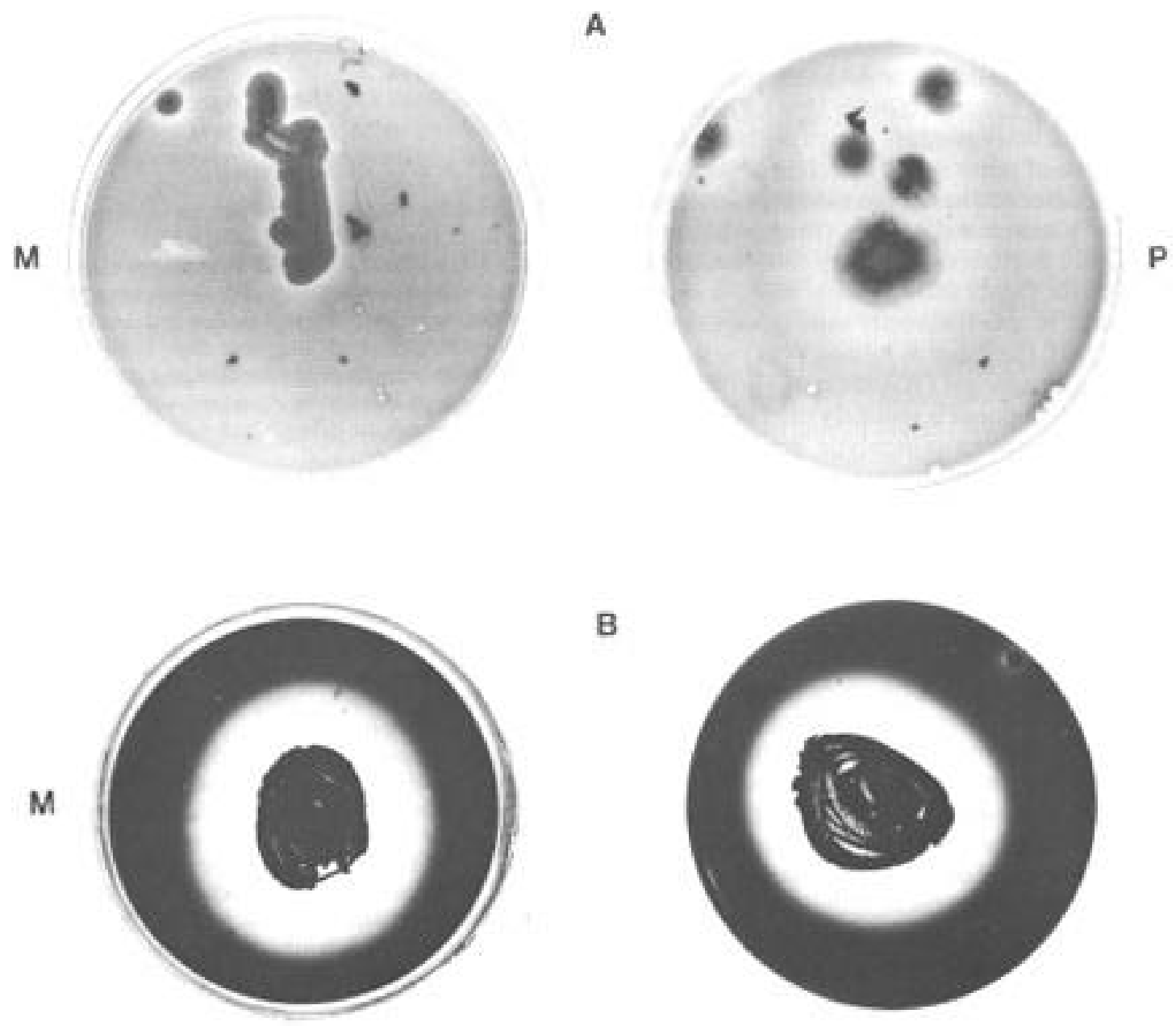

B

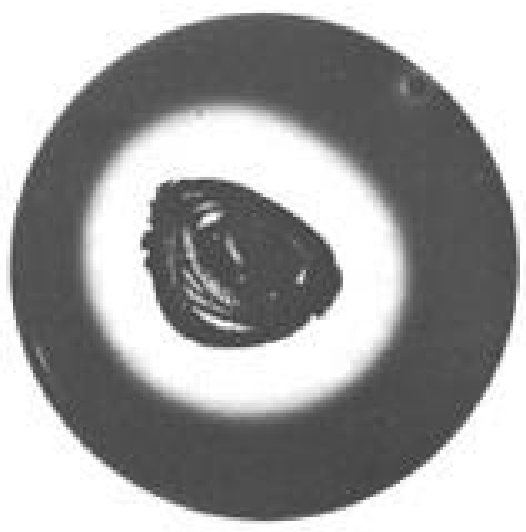

1

2

Fig. 3: A - Production of extracellular proteases in agar plates containing hemoglobin as substrate and BHI-agar as culture medium. M: Micrococcus luteus, P: Pseudomonas aeruginosa. B - Effect of glycerol in protease production by M. luteus. 1: medium C, 2: medium D.

inducing substrate, followed by hemoglobin. It is possible that gelatin, as a high molecular weight protein, induces an increase in the protease production to degrade the substrate to an available form for the microorganism.

It has been described that addition of glucose to various media increase the growth of $P$. aeruginosa but there was a marked reduction in the amount of proteinase production. These results suggest that protease production by $P$. aeruginos was subject to catabolite repression by glucose when peptones were the source of nitrogen (Juffs 1976). The composition of the two culture media used in our study (BHI or yeast extract) did not modify the degradation of the substrates, except by $M$. luteus, in which no protease was detected in yeast extract-gelatin and a strong activity was found in BHI-gelatin. Our results showed that M. luteus did not produce protease when sucrose was used as carbon source. The enzyme production is induced when glycerol was used as carbon source (culture media $\mathrm{C}$ and $\mathrm{D}$ ). These results suggest the possibility that sucrose could exert the same effect in catabolite repression than glucose in $P$. aeruginosa.

It has been described methods to assay proteolytic activity involving colorimetric analysis (Rinderknecht et al. 1968), the use of fluorogenic substrate (Rust et al. 1994), zymographic techniques (Lantz \& Ciborowski 1994) and incorporation of different substrates in nutrient agar plates, such as skim milk (Sacherer et al. 1994), BSA (Rüchel et al. 1982), casein (Ahearn et al. 1968) and elastin (Rust et al. 1994). 
In this work, gelatin, BSA and hemoglobin have been used as enzyme substrate. A wide range of methods are available for detecting proteases using gelatin as substrate (Grubb 1994). These methods as with other protease assays are based on the hydrolysis of substrate for detection of gelatinaselike proteases and have been used in several methods for biochemical studies (Branquinha et al. 1996) and in clinical laboratory for identification of bacteria (MacFaddin 1980). BSA, a smaller molecule, is also frequently used incorporated in SDS-PAGE (Kelleher \& Juliano 1984) or in radial diffusion in agar gel (Wikström et al. 1981). Denatured hemoglobin is routinely used in assay systems for detecting proteolytic activity (Anson 1939) and also incorporated in SDS-PAGE (Andary \& Dabich 1974).

The technique we describe is of value for detecting extracellular proteases directly in the culture medium. The use of gelatin in the culture medium provided us a qualitative assay, a simple, inexpensive, straight forward method to assess the presence of proteolytic activity of a given colony. In addition, the method allows greater freedom in substrate selection since low-(BSA, hemoglobin) or highmolecular weight (gelatin) proteins could be used. This method, for instance, allows to verify in an initial screening whether the bacteria secrete extracellular proteases and the best culture medium composition to detect this production. In summary, the simplicity of this method is its greatest advantage, although it cannot be used to quantitate degradation.

\section{REFERENCES}

Ahearn DG, Meyers SP, Nichols RA 1968. Extracellular proteinases of yeasts and yeast-like fungi. Appl Microbiol 16: 1370-1374.

Andary TJ, Dabich D 1974. A sensitive polyacrylamide disc gel method for detection of proteinases. Anal Biochem 57: 457-466.

Anson ML 1939. The estimation of pepsin, trypsin, papain and cathepsin with hemoglobin. $J$ Gen Physiol 22: 79-89.

Bhosale SH, Rao MB, Deshpande VV, Srinivasan MC 1995. Thermostability of high activity alkaline protease from Conidiobolus coronatus (NCL 86.8.20). Enzyme Microbiol Technol 17: 136-139.

Bilinski CA, Russel J, Stewart GG 1987. Applicability of yeast extracellular proteinases in brewing: physiological and biochemical aspects. Appl Environm Microbiol 53: 495-499.

Branquinha MH, Vermelho AB, Goldenberg S, Bonaldo MC 1996. Ubiquity of cysteine- and metalloproteinase activities in a wide range of trypanosomatids. J Euk Microbiol 43: 131-135.

Brown PD 1994. Clinical trials of a low molecular weigh matrix metalloproteinase inhibitors in cancer. Inhibition of matrix metalloproteinases. Therapeutic potencial. An NY Acad Sci 732: 217-221.

de Giorgi GS, de Valdez GF, de Ruiz Itolgada AP, Oliver
G 1985. Effect of $\mathrm{pH}$ and temperature on the proteolytic activity of lactic acid bacteria. $J$ Dairy Sci 68: 2160-2164.

Fujishige A, Smith KR, Silen JL, Agard DA 1992. Correct folding of a-lytic protease is required for its extracellular secretion from Escherichia coli. J Cell Biol 118: 33-42.

Grubb JD 1994. Assay for bacterial type I collagenases. Methods Enzymol 235: 602-606.

Heussen C, Dowdle EB 1980. Electrophoretic analysis of plasminogen activators in polyacrylamide gels containing sodium dodecyl sulfate and copolymerized substrates. Anal Biochem 102: 196-202.

Juffs HS 1976. Effects of temperature and nutrients on proteinase production by Pseudomonas fluorescens and $P$. aeruginosa in broth and milk. $J$ Appl Bact 40: $23-32$.

Kelleher PJ, Juliano RL 1984. Detection of proteases in polyacrylamide gels containing covalently bound substrates. Anal Biochem 136: 470-475.

Lantz MS, Ciborowski P 1994. Zymographic techniques for detection and characterization of microbial proteases. Methods Enzymol 235: 563-594.

Mac Fadin JF 1980. Pruebas bioquimicas para la identification de bacterias de importancia clinica. The Williams and Wilkins Company-Baltimore.

Maeda H, Morihara K 1995. Serralysin and related bacterial proteinases. Methods Enzimol 248: 395-413.

Morihara K 1995. Pseudolysin and other pathogen endopeptidases of thermolysin family. Methods Enzimol 248: 242-253.

Petinate SDG, Meirelles MNL, Branquinha MH, Chaia AA, Ferreira MN, Vermelho AB 1995. Micrococcus luteus - protein, glycoconjugate and proteolytic profiles. XXIV Reunião Anual da Sociedade Brasileira de Bioquímica e Biologia Molecular, Caxambu, MG, p.142.

Ponsare AC, Venugopai V, Lewis NF 1985. A note on nutritional influence on extracellular protease synthesis in Aeromonas hydrophila. J Appl Bacteriol 58: 101-104.

Rinderknecht H, Geokas NC, Silverman P, Haverback BJ 1968. A new ultrasensitive method for the determination of proteolytic activity. Clin Chem Acta 21: 197-203.

Rüchel R, Tegeler R, Trost M 1982. A comparison of secretory proteinases from different strains of Candida albicans. Sabouraudia 20: 233-244.

Rust L, Messing CR, Iglewski BH 1994. Elastase assays. Methods Enzymol 235: 555-562.

Sacherer P, Défago G, Haas D 1994. Extracellular protease and phospholipase $\mathrm{C}$ are controlled by the global regulatory gene gac A in the biocontrol strain Pseudomonas fluorescens $\mathrm{CHaO}$. FEMS Microbiol Lett 116: 155-160.

Sakata K, Yajima H, Tanaka K, Sakamoto Y, Yamamoto K, Yoshida A, Dohs Y 1993. Erythromycin inhibits the production of elastase by Pseudomonas aeruginosa without affecting its proliferation in vitro. Am Rev Respir Dis 148: 1061-1065.

Wikström M, Elwing H, Linde A 1981. Determination of proteolytic activity: A sensitive and simple assay utilizing substrate adsorbed to a plastic surface and radial diffusion in gel. Anal Biochem 118: 240-246. 